\title{
Dietary, Anthropometric, Biochemical and Psychiatric Indices in Shift Work Nurses
}

\author{
Mahshid Naghashpour ${ }^{1,2}$, Reza Amani ${ }^{2,3^{*}}$, Sorur Nematpour ${ }^{4}$, Mohammad Hosein Haghighizadeh ${ }^{5}$ \\ ${ }^{1}$ Student Research Committee, Ahvaz Jundishapur University of Medical Sciences, Ahvaz, Iran; ${ }^{2}$ Department of Nutrition, School of \\ Paramedicine, Ahvaz Jundishapur University of Medical Sciences, Ahvaz, Iran; ${ }^{3}$ Diabetes Research Center, Ahvaz Jundishapur Uni- \\ versity of Medical Sciences, Ahvaz, Iran; ${ }^{4}$ Golestan Medical Center, Jundishapur University of Medical Sciences, Ahvaz, Iran; \\ ${ }^{5}$ Department of Statistics and Epidemiology, Jundishapur University of Medical Sciences, Ahvaz, Iran. \\ Email: "rezaamani@hotmail.com
}

Received June $13^{\text {th }}, 2013$; revised July $13^{\text {th }}, 2013$; accepted July $20^{\text {th }}, 2013$

Copyright (C) 2013 Mahshid Naghashpour et al. This is an open access article distributed under the Creative Commons Attribution License, which permits unrestricted use, distribution, and reproduction in any medium, provided the original work is properly cited. In accordance of the Creative Commons Attribution License all Copyrights (C) 2013 are reserved for SCIRP and the owner of the intellectual property Mahshid Naghashpour et al. All Copyright (C) 2013 are guarded by law and by SCIRP as a guardian.

\begin{abstract}
Objectives: The aim of this study was to compare nutrients intake, anthropometric, biochemical and psychiatric indices between shift working and day-time nurses. Methods: A cross-sectional comparative study was conducted in which ninety eight female nurses (55 day-time workers and 43 shift workers) from six educational hospitals of Jondi-Shapour University of medical sciences, Ahvaz, Iran were participated. A questionnaire including dietary, anthropometric, disease history and lifestyle pattern questions was completed and 3-day 24-hour recalls, Beck Depression Inventory (BDI) and Hospital Anxiety and Depression Scale (HADS) were collected. Serum hs-CRP and $25(\mathrm{OH}) \mathrm{D}_{3}$ concentrations were measured by immunoturbidimetric and electrochemiluminescent immunoassay method, respectively. Anthropometric indices were measured according to World health organization standard protocol. Independent sample $t$ and chi-square tests were used for statistical analysis. Results: There was a lower dietary intake of thiamin, riboflavin, niacin, folate, magnesium and iron in shift worker compared with day-time nurses $(p<0.05)$. No significant differences in serum hs-CRP concentrations, serum $25(\mathrm{OH}) \mathrm{D}_{3}$ levels, vitamin D deficiency percentage, hemoglobin and hematocrite concentrations, and also anthropometric and psychiatric variables were found between two groups. Duration of exposure to sunlight was significantly higher in shift workers than in day time nurses. Engagement time in weekly physical exercise was around 11 times greater in day-time nurses compared with the shift work nurses $(p=0.001)$. Conclusions: This study showed that shift working is associated with some nutritional deficiencies and sedentary lifestyle among female nurses.
\end{abstract}

Keywords: Shift Work; Dietary Intake; Depression; Nurse

\section{Introduction}

Shift work generally is defined as "work hours that are scheduled outside of daylight" [1]. Nurses represent an important part of the hospital workforce and serve as role models when caring for patients [2]. According to a study, night working is considered as a kind of challenge among most nurses and can lead to several forms of physical and emotional disorders [3]. Shift duties were positively associated with abnormal eating habits among hospital nurses. Studies on the effects of shift work on eating habits and nutrients intake have previously been

\footnotetext{
${ }^{*}$ Corresponding author.
}

conducted on shift workers in different jobs [4,5]. Shift workers preferred to eat cold and fast foods and tend to have a nibble rather than a meal and have fewer meals over 24 hours [5]. These eating habit changes may increase or decrease intake of nutrients. Shift work and insufficient rest, as both are considered the main obstacle to healthy eating by respondents. Obesity has been shown to be more prevalent among shift workers and is associated with higher BMI, independently of age and work duration $[5,6]$. Several mechanisms explain weight gain in shift workers, such as higher calorie intake, changes in dietary habits such as eating fewer meals, more snacks and in the circadian distribution of food 
intake and lower physical exercise [5]. Modifying external factors such as food and beverage intake patterns can help to reduce the destructive health effects of shift work [1]. Compared to the number of studies that evaluated intra-individual differences in nutrient intake by shift, there have been few studies that have examined the difference in micronutrient intakes between shift workers and day time workers [5].

Findings highlight that night and early morning working is associated with an adverse profile of cardiovascular risk factors, which are partly explained by socioeconomic, other occupational factors, and health behaviors [7]. Moreover, shift work leads to change in lifestyle, for instance in relation to diet, smoking, alcohol and exercise, all being risk factors of ischemic heart disease [8]. We measured the high-sensitivity C-reactive protein (hsCRP) which is being increasingly used as a marker for cardiac risk assessment and as a prognostic tool in heart disease [9].

Studies have demonstrated that daylight exposure may be effective on job burnout in nurses. Daylight at least 3 $\mathrm{h}$ a day was found to cause less stress and higher satisfaction at work in them [10]. A more relaxed and less tense mood has been reported after UV exposure compared with after non UV exposure [11]. In addition, decreased 25-hydroxyvitamin D is associated with Depression [12, 13]. Since the shift workers presumably have a poor sun exposure and a higher risk of vitamin D deficiency, we have also measured vitamin D status in nurses.

To our best knowledge, there is no other study published concerning the effect of shift work on daily nutriaents intake, anthropometric, biochemical and psychiatric indices of nurses. This study was aimed to compare these indices between shift work and daytime nurses in University hospitals.

\section{Material and Methods}

\subsection{Study Design and Sample}

In this analytical cross-sectional comparative study, healthy female clinical nurses with the mean age of 37 years old (range from of 23 years to 52 years old) employed in educational University hospitals in Ahvaz city, Iran, participated in April to September 2009. Sample size was calculated from a formula

$\left[n=\left(Z_{1-\alpha / 2}+Z_{1-\beta}\right)^{2}\left(\delta_{1}^{2}+\delta_{1}^{2}\right) /\left(\mu_{1}-\mu_{2}\right)^{2}\right] \quad[14] . \alpha$ is type 1 statistical error and $\beta$ is type 2 statistical error. where $n$ is the sample size, $Z_{1-\alpha / 2}$ is the $1-\alpha / 2$ critical value of the standard normal distribution which is found in the table of the standard normal distribution, $\delta$ is the known sample standard deviation, and $\mu$ is the sample mean. A three-stage sampling design was used. At each stage, simple random sampling was applied. In the first stage of sampling, the sampling unit was educational University hospitals (administrative units). A total of six hospitals were randomly selected from eight hospitals. In the second stage, on average, from a total of 1350 nurses, 20 volunteers were selected randomly from each sample hospital. A total of 120 volunteers were selected. A selfreported questionnaire assessed demographic characteristics, disease history (chronic and psychiatric), medication and supplement usage, pregnancy, smoking status and physical activity, were completed by volunteers. Subjects with the history of anemia, thyroid disorders, renal failure, heart failure, diabetes mellitus, malabsorption, chronic and psychiatric disease, pregnancy, history of using medicines and other substances that could affect appetite were excluded from this study. 22 individuals who were not eligible were excluded. In the third stage, 98 volunteers who met inclusion criteria were categorized in day time and shift workers groups (55 day time workers and 43 shift workers).

Shift work was defined as work at times out of normal daylight hours 8:00 am to 2:00 pm or work during the weekends [15]. An additional question representing the shift work period was asked. In this study, nurses were divided into two groups: shift-work and day-time nurses.

Participants completed a questionnaire included demographic characteristics, physical activity during the week, duration of exposure to sunlight and use of tobacco over a three-month period.

The study protocol was approved by the research ethics committee of Ahvaz Jondi-Shapour University of Medical Sciences (AJUMS). Written consents were obtained from all participants.

\subsection{Assessment of Depression and Anxiety}

Depression status was assessed using the short 13-items version Beck Depression Inventory (BDI) and total scores (BDI score) were calculated. The BDI cut-off values $\geq 5$ is defined as depression [16]. The BDI included questions to assess nine characteristic attitudes and symptoms listed in the DSM-IV criteria for a major depressive episode. Anxiety was evaluated by the Hospital Anxiety and Depression Scale (HADS). HADS anxiety score between $8-10$ and $11-21$ were considered as mild and clinical anxiety, respectively [17].

\subsection{Dietary Assessment}

We assessed dietary intake using a 3-day 24-hour recall (including 2 working days and one off-day). Quantities were expressed in household measures. The inventories were double checked by a nutritionist, who verified the quantities and type of recalled foods. Daily dietary intakes of energy and micronutrients (thiamin, riboflavin, niacin, pyridoxine, cobalamin, folate, ascorbate, toco- 
pherol, retinol, cholecalciferol, magnesium, zinc, iron and calcium) were analyzed using Iranian Food Processor software, which has been developed by the Iranian Institute of Nutrition Research and Food Industry and comprises Iranian meals dataset.

\subsection{Biochemical Measurements}

Fasting blood samples were obtained into potassium EDTA - containing tubes for determination of hemoglobin and hematocrite and tubes with no anticoagulants for serum hs-CRP and $25(\mathrm{OH}) \mathrm{D}_{3}$ levels evaluation. Determination of Hemoglobin was done by Cyanmethemoglobin Method and Hematocrit by calculating the percentage of blood that is comprised of red blood cell (RBC) [18].

Serum hs-CRP level were measured by a particle-enhanced turbidimetric immunoassay (quantitative diagnosis kit for serum or plasma hs-CRP by immunoturbidimetric method; Pars Azmoon, Tehran, Iran) with a limit of detection of $0.10 \mathrm{mg} / 1$ [19]. In this study, the Risk Evaluation Guidelines proposed by American Heart Association and Center for Disease Control and prevention (AHA/CDC) was used for categorizing hs-CRP levels [9].

Serum 25(OH)D level were measured during summer months using Electrochemiluminescent immunoassay [20] (Serum 25(OH)D kit; Roche, Britain). Lips [21] cut-offs were used for vitamin D deficiency evaluation which has categorized vitamin D deficiency based on increasing serum parathormon (PTH) levels and bone histology. In this method, values 25 - 50, 12.5 - 25 and < $12.5 \mathrm{nmol} / 1$ indicate mild (insufficiency), moderate and severe vitamin $\mathrm{D}$ deficiency, respectively.

\subsection{Anthropometric Indices}

Anthropometric indices including weight and height indices were measured according to WHO (World health organization) standard protocol [22]. Body Mass Index (BMI), Body Fat Percent (\% BF) and Fat to Weight Ratio (F/W) were assessed by bioelectric impedance methods (Omron, BF 302, Japan).

\subsection{Statistical Analysis}

Independent sample $t$ and chi-square tests were carried out for analyzing the quantitative and categorized variables, respectively. $p$ values less than 0.05 were regarded as significant. SPSS software version 13 was used for statistical analysis.

\section{Results}

Table 1 shows the basic demographic characteristics of participations in both groups. Results show that there is no significant difference between shift and day-time
Table 1. The basic demographic characteristics of participations.

\begin{tabular}{cccc}
\hline \multirow{2}{*}{$\begin{array}{c}\text { Basic demographic } \\
\text { characteristics }\end{array}$} & \multicolumn{3}{c}{ Study groups } \\
\cline { 2 - 4 } & $\begin{array}{c}\text { Shift workers } \\
(\mathrm{N}=43)\end{array}$ & $\begin{array}{c}\text { Day time workers } \\
(\mathrm{N}=55)\end{array}$ & p value \\
\hline Age (Year) $)^{\mathrm{a}}$ & $33 \pm 5.3$ & $33.7 \pm 3.3$ & 0.963 \\
Married status $^{\mathrm{b}}$ : Married & $74.4(32)$ & $73.6(39)$ & 0.865 \\
Work experience & $14.7 \pm 5.1$ & $15.8 \pm 5.3$ & 0.753 \\
Overtime work $^{\mathrm{b}}$ yes & $44.2(19)$ & $32.7(18)$ & 0.112 \\
\hline
\end{tabular}

${ }^{\mathrm{a}}$ Quantitative data presented as mean $\pm \mathrm{SD}$. Independent sample t-test was conducted; ${ }^{\text {b }}$ ualitative data presented as percent (number). Chi square test was conducted.

worker nurses in terms of obtained demographic variables.

\subsection{Comparison of Nutrients Intakes}

Table 2 compares total energy and dietary micronutrient intakes between shift workers and day time workers. Lower dietary intakes of thiamin, riboflavin, niacin, folate, magnesium and iron were seen in shift workers compared with the day-time nurses. There were no significant differences in other micronutrient and energy intakes between these two groups.

\subsection{Comparison of Biochemical Variables}

Table 3 shows values for biochemical variables in both shift work and day time nurses. No significant differences were found in serum hs-CRP concentrations and percentage of risk measurements between shift work and daytime nurses. Serum $25(\mathrm{OH}) \mathrm{D}_{3}$ levels $(\mathrm{nmol} / \mathrm{l})$, vitamin D deficiency severity percent and hematologic indices were not significantly different between two groups.

\subsection{Comparison of Anthropometric, Lifestyle and Psychiatric Indices}

Table 4 indicates that there is no significant difference in anthropometric variables between two groups. Nor BDI neither HADS anxiety scores revealed any statistically significant differences between two groups. Duration of daily exposure to sunlight was significantly higher in shift workers $(p=0.013)$ compared with day time nurses. Duration of weekly physical exercise was more than eleven times higher in daytime nurses compared with the shift workers $(p=0.001)$.

\section{Discussion}

According to our search, this was the first study carried out to assess the dietary intake, anthropometric, biochemical, lifestyle and psychiatric parameters in hospital shift working nurses. 
Table 2. The comparison of dietary nutrients intakes (Mean \pm SD) between shift work and day-time nurses ${ }^{\mathrm{a}}$.

\begin{tabular}{|c|c|c|c|}
\hline \multirow{2}{*}{ Dietary intakes } & \multicolumn{3}{|c|}{ Study groups } \\
\hline & Shift workers $(\mathrm{N}=43)$ & Day time workers $(\mathrm{N}=55)$ & $p$ value \\
\hline Thiamin (mg/day) & $1.2 \pm 0.55$ & $1.6 \pm 1.2$ & $0.01^{\mathrm{b}}$ \\
\hline Riboflavin (mg/day) & $1.6 \pm 0.72$ & $2.1 \pm 1.1$ & $0.003^{\mathrm{b}}$ \\
\hline Niacin (mg/day) & $16 \pm 7.7$ & $21.1 \pm 13.1$ & $0.01^{\mathrm{b}}$ \\
\hline Pyridoxine (mg/day) & $1.6 \pm 0.82$ & $1.1 \pm 1$ & 0.71 \\
\hline Cobalamin ( $\mu \mathrm{g} /$ day $)$ & $3.6 \pm 2.3$ & $5.2 \pm 6$ & 0.1 \\
\hline Folate ( $\mu \mathrm{g} /$ day) & $284.7 \pm 157.4$ & $386 \pm 236.8$ & $0.007^{\mathrm{b}}$ \\
\hline Ascorbate (mg/day) & $117.6 \pm 76$ & $160.9 \pm 204.7$ & 0.18 \\
\hline Tocopherol (mg/day) & $7.9 \pm 4.6$ & $12.8 \pm 33.38$ & 0.16 \\
\hline Retinol ( $\mu \mathrm{g} /$ day) & $947.3 \pm 663.9$ & $1319.6 \pm 906.3$ & 0.11 \\
\hline Cholecalciferol ( $\mu \mathrm{g} /$ day) & $2.4 \pm 4.4$ & $2.42 \pm 2.1$ & 0.56 \\
\hline Magnesium (mg/day) & $251.7 \pm 76.3$ & $317.4 \pm 208.75$ & $0.049^{\mathrm{b}}$ \\
\hline Zinc (mg/day) & $8 \pm 2.6$ & $10.1 \pm 4.7$ & 0.09 \\
\hline Iron (mg/day) & $11.8 \pm 4.8$ & $15.3 \pm 9.5$ & $0.03^{\mathrm{b}}$ \\
\hline Calcium (mg/day) & $862.9 \pm 313.5$ & $940.7 \pm 488.3$ & 0.18 \\
\hline Energy intake (kcal/day) & $1637.1 \pm 542.6$ & $1859.7 \pm 539.8$ & 0.43 \\
\hline
\end{tabular}

${ }^{\mathrm{c}}$ Independent sample t-test was conducted; ${ }^{\mathrm{d}}$ Lower dietary intakes of thiamin, riboflavin, niacin, folate, magnesium and iron were seen in shift works compared with the day-time nurses $(p<0.05)$.

Table 3. The comparison of biochemical indices between shift work and day-time nurses ${ }^{\mathrm{a}}$.

\begin{tabular}{|c|c|c|c|}
\hline \multirow{2}{*}{ Biochemical variables } & \multicolumn{3}{|c|}{ Study groups } \\
\hline & Shift workers $(\mathrm{N}=43)$ & Day-time workers $(\mathrm{N}=55)$ & $p$ value \\
\hline hs-CRP serum levels $(\mathrm{mg} / \mathrm{l})^{\mathrm{b}}$ & $2.1 \pm 2.3$ & $2.21 \pm 2.6$ & 0.89 \\
\hline \multicolumn{4}{|l|}{ percent of risk measurements $^{c}$} \\
\hline$<1 \mathrm{mg} / 1$ (low risk) & $46.5(20)$ & $42.3(22)$ & 0.72 \\
\hline $1-3$ mg/l (medium risk) & $32.6(14)$ & $40.4(21)$ & \\
\hline$>3 \mathrm{mg} / \mathrm{l}$ (high risk) & $20.9(9)$ & $17.3(9)$ & \\
\hline Serum 25(OH)D3 levels $(\mathrm{nmol} / \mathrm{l})^{\mathrm{b}}$ & $26.3 \pm 20.5$ & $27.4 \pm 22.7$ & 0.39 \\
\hline \multicolumn{4}{|l|}{ Vitamin D deficiency severity $^{c}$} \\
\hline Severe & $39.5(17)$ & $39.6(21)$ & 0.88 \\
\hline Moderate & $18.6(8)$ & $13.2(7)$ & \\
\hline Mild & $30.2(13)$ & $35.8(19)$ & \\
\hline Normal & $11.6(5)$ & $11.3(6)$ & \\
\hline Hemoglobin $(g / l)^{b}$ & $11.5 \pm 0.92$ & $11.16 \pm 1$ & 0.97 \\
\hline Hematocrite $(\%)^{\mathrm{b}}$ & $37.3 \pm 4.3$ & $36.1 \pm 2.8$ & 0.67 \\
\hline
\end{tabular}

${ }^{\mathrm{a}}$ There was no significant difference between shift works and day-time nurses in biochemical indices; ${ }^{\mathrm{b}}$ Quantitative data presented as mean \pm SD. Independent sample t-test was conducted to analyze data; ${ }^{\mathrm{C}}$ Qualitative data presented as percent (number). Chi square test was conducted. 
Table 4. The comparison of anthropometric, lifestyle and psychiatric indices between shift work and day-time nurses.

\begin{tabular}{|c|c|c|c|}
\hline \multirow{2}{*}{ Indices } & \multicolumn{3}{|c|}{ Study groups } \\
\hline & Shift workers $(\mathrm{N}=43)$ & Day-time workers $(\mathrm{N}=55)$ & $p$ value \\
\hline \multicolumn{4}{|l|}{ Anthropometric variables } \\
\hline Weight $(\mathrm{kg})^{\mathrm{a}}$ & $64 \pm 11.8$ & $66.6 \pm 11.1$ & 0.55 \\
\hline Body fat percent $(\% \mathrm{BF})^{\mathrm{a}}$ & $29.8 \pm 6.5$ & $32.27 \pm 6.3$ & 0.84 \\
\hline Fat to Weight Ratio (F/W) & $20.7 \pm 8$ & $23.1 \pm 88.1$ & 0.72 \\
\hline Body Mass Index (BMI) $\left(\mathrm{kg} / \mathrm{m}^{2}\right)^{\mathrm{a}}$ & $25.4 \pm 4.6$ & $26.2 \pm 4.4$ & 0.95 \\
\hline Underweight & $10.8(4)$ & $2.1(1)$ & 0.19 \\
\hline Normal weight $^{\mathrm{b}}$ & $51.4(19)$ & $46.8(22)$ & \\
\hline Over weight $^{\mathrm{b}}$ & $32.4(12)$ & $48.9(23)$ & \\
\hline Obesity $^{\mathrm{b}}$ & $5.4(2)$ & $2.1(1)$ & \\
\hline \multicolumn{4}{|l|}{ Life style variables } \\
\hline Duration of weekly physical exercise (min/wk) & $6.2 \pm 21.7$ & $68.7 \pm 208.36$ & $0.001^{\mathrm{d}}$ \\
\hline Duration of daily exposure to sunlight (min/day) $)^{\text {ad }}$ & $27.3 \pm 33.1$ & $16.39 \pm 19.1$ & $0.013^{\mathrm{e}}$ \\
\hline$<30(\mathrm{~min} / \mathrm{wk})^{\mathrm{b}}$ & $61(25)$ & $71.2(37)$ & 0.23 \\
\hline $30-60(\mathrm{~min} / \mathrm{wk})^{\mathrm{b}}$ & $19.5(8)$ & $21.2(11)$ & \\
\hline$>60(\mathrm{~min} / \mathrm{wk})^{\mathrm{b}}$ & $19.5(8)$ & $7.7(4)$ & \\
\hline \multicolumn{4}{|l|}{ Psychiatric variables } \\
\hline BDI score ${ }^{a}$ & $4.79 \pm 3.35$ & $3.65 \pm 3.64$ & 0.32 \\
\hline \multicolumn{4}{|l|}{ Depression severity ${ }^{\mathrm{b}}$} \\
\hline Mild & $37.2(16)$ & $21.8(12)$ & 0.17 \\
\hline Moderate & $18.6(8)$ & $16.4(9)$ & \\
\hline Severe & $0(0)$ & $0(0)$ & \\
\hline Normal & $44.2(19)$ & $61.8(34)$ & \\
\hline HADS anxiety score ${ }^{a}$ & $4.88 \pm 3.24$ & $3.82 \pm 3.26$ & 0.58 \\
\hline \multicolumn{4}{|l|}{ Anxiety severity ${ }^{\mathrm{b}}$} \\
\hline Normal & $78.6(33)$ & $87.3(48)$ & 0.48 \\
\hline Moderate & $14.3(6)$ & $7.3(4)$ & \\
\hline Clinical & $7.1(3)$ & $5.5(3)$ & \\
\hline
\end{tabular}

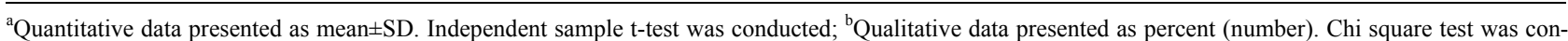
ducted; ${ }^{\mathrm{c}}$ Duration of weekly physical exercise was more than eleven times higher in daytime nurses compared with the shift works; ${ }^{\mathrm{d}}$ Duration of daily exposure to sunlight was significantly higher in shift workers compared with daytime nurses.

This study showed that shift working nurses had lower dietary intakes of some B vitamins (including thiamin, riboflavin, niacin and folate), two trace elements magnesium and iron compared with daytime nurses. Higher duration of daily exposure to sunlight and lower duration of weekly physical exercise were also observed in the shift working group.

There are few studies investigating the effect of shift working on dietary nutrients intake mostly focused on dietary habits. Some authors did not find any difference between shift workers and day time employees with respect to their total energy intake and dominant macronutrient intakes [5]. Morikawa have reported that Japanese shift workers, particularly the late-shift workers, took smaller amounts of energy and nutrients than the daytime workers. Their inadequate nutrient intake was due to 
lower meal frequency and poor meal quality, both of which were conditioned by shift work. One study on the impact of work hours on eating habits and dietary intake of male industrial workers in day workers and two- and three-shift workers, showed no changes in intake of energy, nutrients and coffee/tea between 8 hour morning and afternoon shifts, but a reduction in intake of nutrients and coffee/tea during 8 hours night shifts. On the whole, the energy-intake and the quality of food intake (percentages of energy from macronutrients and density of micronutrients) were not affected by shift work [5].

In recent study on nurses in Hong Kong [4], shift duties were positively associated with abnormal eating behavior among nurses working in hospitals. It has been reported that the total energy and nutrient intakes increase when individuals eat with others [23]. However shift workers have fewer opportunities to take meals with their families and friends. These effects appeared to be most prominent among workers with midnight shifts, who decreased their micronutrients intakes [5].

In Morikawa et al. study [5], the energy density value of thiamin, vitamin A and potassium in shift workers with mid night shifts were significantly lower than those with fixed day workers. Also, in this study, energy intake of dairy products (one of the good sources of riboflavin), meat (good sources of niacin, folate and iron) and vegetables (rich in riboflavin, folate and magnesium) were the lowest in shift workers with mid night shift than fixed day workers. These results are comparable with our findings on nutrients intake of shift nurses. Several studies agree that shift work affects the distribution of food intake and the selection of food items over 24 hour $[24,25]$.

In this study, it is suggested that subjects had undesirable food items selection of that can result in long term complications of micronutrients deficiencies. Such deficiencies can lead to impaired energy and macronutrients metabolism and synthesis, immune system dysfunction, anorexia, neurologic and cardiac signs, depression, confusion, sleeplessness, fatigue, general weakness, indigestion, osteoporosis and anomies [26].

There was no significant difference between shift and day-time nurses in terms of anthropometric variables. The effect of shift work on body weight has been investigated in previous studies, several of which demonstrated a significant tendency to become overweight $[6$, $27,28]$. In contrast, some studies failed to show such associations $[15,29]$.

According to studies, body fat distribution indices that are body fat percent (\% BF) and Fat to Weight Ratio $(\mathrm{F} / \mathrm{W})$ variables in our study were not different between two shift workers. Weight gain in shift workers has been explained by several mechanisms, such as higher calorie intake, changes in dietary habits (such as eating fewer meals and more snacks) and the circadian distribution of food intake [5,6], as well as lower physical exercise and changes in sleeping habits [5].

In this study, there was no significant difference in total energy intake between shift workers and day time workers whereas, in most of the other studies mentioned above, no clear-cut conclusion in this respect was presented.

According to our results, duration of daily exposure to sunlight was significantly higher in shift workers. Night shift workers can improve nocturnal alertness and daytime sleep by bright light exposure in their work place. These improvements can be maximized by attenuating morning light on the way home. Melatonin exerted modest benefit in improving the adaptation of workers to nightshift, and its effect was not enhanced by attenuation of morning sunlight exposure [30]. This result may, in part, explain the nonsignificant findings of psychiatric variables due to higher duration of exposure time to sun light.

In our study, duration of weekly physical exercise was significantly lower in shift nurses compared with day time nurses. In an interventional study on shift workers, overall "disengagement" coping scores from the SSI (Standard Shift-work Index) were positively related to leisure-time energy expenditure [31]. In another study lack of exercise was the lifestyle factor which strongly contributed to chronic fatigue in shift working nurses [32]. Time spent in shift work was found to be predictors of energy expenditure. It is recommended that physical activity interventions for shift workers should be designed with careful consideration of individual domestic responsibilities and perceived disruption to sleep [32]. Concerning the rhythm desynchronization of transfering from shift work to day work, it has been suggested that while examining tolerance/in tolerance of a shift worker to rotational shift work, the levels of anxiety and mental health status of the individual under careful and thorough examination should be taken into consideration. In this study, there was no significant difference in serum hsCRP concentrations and percentage of risk measurements between shift working and daytime workers. Serum $25(\mathrm{OH}) \mathrm{D}_{3}$ levels (nmol/litr), vitamin D deficiency severity percent, hemoglobin and hematocrite concentrations were not different between two groups. It is suggested that these insignificant findings are resulted from limited numbers of subjects in each group. In this study, one of the Limitations was the descriptive comparative nature of its design. The other limitation was that laboratory measurements of micronutrients were not used to assess nutritional status. There for, it is suggested that in future studies evaluation of micronutrients status by proper biochemical methods would confirm our results. In summary, shift duties were associated with unhealthy 
lifestyle and nutritional imbalances accompanied by deficiencies of some B vitamin, magnesium and iron intakes among hospital shift nurses. More health promotional initiatives and education should be targeted towards hospital nurses whose duties require frequent shifts to enhance healthier eating and lifestyle patterns. In this study, it is recommended that in every work place where shift work is mandatory, trained health care personnel should monitor intermittently (preferably every alternate year) the state of the psychological and nutritional status of each shift nurse to minimize the occupational health hazards and enhance their performance.

\section{Conclusions}

As a conclusion, shift work is associated with unhealthy lifestyle and nutritional imbalances accompanied by deficiencies of some B vitamin, magnesium and iron intakes among hospital clinical nurses. Moreover, engagement in planned physical activities was lower in shift nurses.

More health promotional initiatives and education programs should be targeted towards nurses whose duties require frequent shifts in order to enhance their eating and lifestyle patterns. It is suggested that in every work place where shift work is mandatory, trained health care personnel should frequently monitor nutritional status of the staff to minimize the occupational health hazards and enhance their performance.

\section{Acknowledgements}

This study was supported by Vice-Chancellor for Research Affairs, Jondi-Shapour University of Medical Sciences as a Grant for the Master of Science Thesis. We wish to thank Dr. Ahmad Zaree Javid (Ph.D) for his kind grammatical edition and Mr. Ahmad Hemadi (MSc.) for his kind laboratory assistance.

\section{REFERENCES}

[1] A. M. Berger and B. B. Hobbs, "Impact of Shift Work on the Health and Safety of Nurses and Patients," Clinical Journal of Oncology Nursing, Vol. 10, No. 4, 2006, pp. 465-471. http://dx.doi.org/10.1188/06.CJON.465-471

[2] J. M. Zapka, S. C. Lemon, R. P. Magner and J. Hale, "Lifestyle Behaviors and Weight among Hospital-Based Nurses," Journal of Nursing Management, Vol. 17, No. 7, 2009, pp. 853-860. http://dx.doi.org/10.1111/j.1365-2834.2008.00923.x

[3] A. N. Nasrabadi, H. Seif, M. Latifi, N. Rasoolzadeh and A. Emami, "Night Shift Work Experiences among Iranian Nurses: A Qualitative Study," International Nursing Review, Vol. 56, No. 4, 2009, pp. 498-503. http://dx.doi.org/10.1111/j.1466-7657.2009.00747.x

[4] H. Wong, M. C. Wong, S. Y. Wong and A. Lee, "The Association between Shift Duty and Abnormal Eating
Behavior among Nurses Working in a Major Hospital: A Cross-Sectional Study," International Journal of Nursing Studies, Vol. 47, No. 8, 2010, pp. 1021-1027. http://dx.doi.org/10.1016/j.ijnurstu.2010.01.001

[5] Y. Morikawa, K. Miura, S. Sasaki, K. Yoshita, S. Yoneyama, M. Sakurai, M. Ishizaki, T. Kido, Y. Naruse, Y. Suwazono, M. Higashiyama and H. Nakagawa, "Evaluation of the Effects of Shift Work on Nutrient Intake: A Cross Sectional Study," Journal of Occupational Health, Vol. 50, No. 3, 2008, pp. 270-278. http://dx.doi.org/10.1539/joh.L7116

[6] L. C. Antunes, R. Levandovski, G. Dantas, W. Caumo and M. P. Hidalgo, "Obesity and Shift Work: Chronobiological Aspects," Nutrition Research Reviews, Vol. 23, No. 1, 2010, pp. 155-168. http://dx.doi.org/10.1017/S0954422410000016

[7] C. Thomas and C. Power, "Shift Work and Risk Factors for Cardiovascular Disease: A Study at Age 45 Years in the 1958 British Birth Cohort," European Journal of Epidemiology, Vol. 25, No. 5, 2010, pp. 305-314. http://dx.doi.org/10.1007/s10654-010-9438-4

[8] A. Knutsson, H. Andersson and U. Berglund, "Serum Lipoproteins in Day and Shift Workers: A Prospective Study," British Journal of Industrial Medicine, Vol. 47, No. 2, 1990, pp. 132-134.

[9] "Inflammation, Heart Disease and Stroke," American Heart Association, 2008.

http://www.americanheart.org/presenter.jhtml?identifier= 4648

[10] M. K. Alimoglu and L. Donmez, "Daylight Exposure and the Other Predictors of Burnout among Nurses in a University Hospital," International Journal of Nursing Studies, Vol. 42, No. 5, 2005, pp. 549-555.

http://dx.doi.org/10.1016/j.ijnurstu.2004.09.001

[11] S. R. Feldman, A. Liguori, M. Kucenic, S. R. Rapp, A. B. Fleischer Jr., W. Lang and M. Kaur, "Ultraviolet Exposure Is a Reinforcing Stimulus in Frequent Indoor Tanners," Journal of the American Academy of Dermatology, Vol. 51, No. 1, 2004, pp. 45-51. http://dx.doi.org/10.1016/j.jaad.2004.01.053

[12] A. Pan, L. Lu, O. H. Franco, Z. Yu, H. Li and X. Lin, "Association between Depressive Symptoms and 25-Hydroxyvitamin D in Middle-Aged and Elderly Chinese," Journal of Affective Disorders, Vol. 118, No. 1-3, 2009, pp. 240-243. http://dx.doi.org/10.1016/j.jad.2009.02.002

[13] W. J. Hoogendijk, P. Lips, M. G. Dik. D. J. Deeg, A. T. Beekman and B. W. Penninx, "Depression Is Associated with Decreased 25-Hydroxyvitamin D and Increased Parathyroid Hormone Levels in Older Adults," Archives of General Psychiatry, Vol. 65, No. 5, 2008, pp. 508-512. http://dx.doi.org/10.1001/archpsyc.65.5.508

[14] M. Naghashpour, R. Amani, S. Nematpour and M. H. Haghighizadeh. "Riboflavin Status and Its Association with Serum hs-CRP Levels among Clinical Nurses with Depression," Journal of the American College of Nutrition, Vol. 30, No. 5, 2011, pp. 340-347. http://dx.doi.org/10.1080/07315724.2011.10719977

[15] M. Ghiasvand, R. Heshmat, R. Golpira, V. Haghpanah, A. Soleimani, P. Shoushtarizadeh, S. M. Tavangar and B. 
Larijani, "Shift Working and Risk of Lipid Disorders: A Cross-Sectional Study," Lipids in Health and Disease, Vol. 5, 2006, pp. 1-9. http://dx.doi.org/10.1186/1476-511X-5-9

[16] H. Kaplan and B. Sadock, "Comprehensive Text Book of Psychiatry," 12th Edition, Lippincott Williams \& Willkins Ltd., USA, 2009, pp. 1047-1049.

[17] A. S. Sigmond and P. Snaiphr, "The Hospital Anxiety and Depression Scale," Acta Psychiatrica Scandinavica, Vol. 67, No. 6, 1983, pp. 361-370. http://dx.doi.org/10.1111/j.1600-0447.1983.tb09716.x

[18] B. J. Bain, S. M. Lewis and I. Bates, "Basic Haematological Techniques," In: S. M. Lewis, B. J. Bain and I. Bates Dacie, Eds., Practical Hematology, 10th Edition, Churchili Livingstone Elsevier Ltd., Philadelphia, 2006, p. 26.

[19] B. Kusnierz-Cabala, W. Gernand, A. Zabek-Adamska, A. Tokarz and J. W. Naskalski, " Comparison of High-Sensitivity C-Reactive Protein Serum Assay Results Obtained Using Dade-Behring BNII Nephelometer and Ortho Vitros FS 5.1 Clinical Analyzer in Respect of CRP-Related Risk Assessment of Chronic Metabolic Diseases," Clinical Laboratory, Vol. 54, No. 9-10, 2008, pp. 341-346.

[20] R. Heshmat, K. Mohammad, S. R. Majdzadeh, M. H. Forouzanfar, A. Bahrami, G. H. Ranjbar Omrani, I. Nabipour, R. Rajabian, A. Hossein-Nezhad, M. Rezaei Hemami, M. Pajouhi and B. Larijani, "Vitamin D Deficiency in Iran: A Multi-Center Study among Different Urban Areas," Iranian Journal of Public Health, a Supplementary Issue on Osteoporosis and Bone Turnover, No. 1, 2008, pp. 72-78.

[21] P. Lips, "Vitamin D Deficiency and Secondary Hyperparathyroidism in the Elderly: Consequences for Bone Loss and Fractures and Therapeutic Implications," Endocrine Reviews, Vol. 22, No. 4, 2001, pp. 477-501. http://dx.doi.org/10.1210/er.22.4.477

[22] World Health Organization (WHO), "Physical Status: The Use and Interpretation of Anthropometry," Tech Rep No. 854, 1995. http://whqlibdoc.who.int/trs/WHO_TRS_854.pdf

[23] J. De Castro, "Socio-Cultural Determinants of Meal Size and Frequency," British Journal of Nutrition, Vol. 77, No. 4, 1997, pp. 39-55. http://dx.doi.org/10.1079/BJN19970103

[24] J. Waterhous, P. Buckley, B. Edwards and T. Reilly,
"Measurements of, and Some Reasons for, Difference in Eating Habits between Night and Day Workers," Chronobiology International, Vol. 20, No. 6, 2003, pp. 10751092. http://dx.doi.org/10.1081/CBI-120025536

[25] N. Nikolova, S. Handjiev and K. Angelova, "Nutrition of Night and Shift Workers in Transports," In: G. Costa, G. Cesana and K. Kogi, Eds., Shift Work: Health Sleep and Performance, Peter Lang Ltd., Frankfort, 1990, pp. 538547.

[26] M. L. Gallager, "The Nutrients and Their Metabolism," In: L. K. Mahan and S. Escott-Stump, Eds., Krausés Food \& Nutrition Therapy, 12th Edition, Saunders, Philadelphia, 2008, pp. 74-78,84-86.

[27] B. Karlsson, A. Knuttsson and B. Lindahl, "Is There an Association between Shift Work and Having a Metabolic syndrome? Results from a Population Based Study of 27485 People," Occupational and Environmental Medicine, Vol. 58, No. 11, 2001, pp. 747-752. http://dx.doi.org/10.1136/oem.58.11.747

[28] K. R. Parkes, "Shift Work and Age as Interactive Predictors of Body Mass Index among Offshore Workers," Scandinavian Journal of Work, Environment \& Health, Vol. 28, No. 1, 2002, pp. 64-71.

[29] B. H. Karlsson, A. K. Knutsson, B. O. Lindahl and L. S. Alfredsson, "Metabolic Disturbances in Male Workers with Rotating Three-Shift Work. Results of the WOLF Study," International Archives of Occupational and Environmental Health, Vol. 76, No. 6, 2003, pp. 424-430.

[30] I. Y. Yoon and B. G. Song, "Role of Morning Melatonin Administration and Attenuation of Sunlight Exposure in Improving Adaptation of Night-Shift Workers," Chronobiology International, Vol. 19, No. 5, 2002, pp. 903-913. http://dx.doi.org/10.1081/CBI-120014571

[31] S. Fullick, C. Grindey, B. Edwards, C. Morris, T. Reilly, D. Richardson, J. Waterhouse and G. Atkinson, "Relationships between leisure-time energy expenditure and individual Coping Strategies for Shift-Work," Ergonomics, Vol. 52, No. 4, 2009, pp. 448-455. http://dx.doi.org/10.1080/00140130802707725

[32] E. Samaha, S. Lal, N. Samaha and J. Wyndham, "Psychological, Lifestyle and Coping Contributors to Chronic Fatigue in Shift-Worker Nurses," Journal of Advanced Nursing, Vol. 59, No. 3, 2007, pp. 221-232. http://dx.doi.org/10.1111/j.1365-2648.2007.04338.x 